\title{
Overview of single-particle nonlinear dynamics
}

\author{
E. Todesco
}

CERN, LHC Division Geneva, CH 1211

\begin{abstract}
We give an overview of the single-particle non linear dynamics in circular accelerators. The main topics are: integration of equations of motion, fast symplectic tracking, dynamic aperture definition, long-term methods, quality factors and lattice optimization. Special emphasis is put on ideas and tools developed during the last decade.
\end{abstract}

\section{INTRODUCTION}

The single-particle nonlinear dynamics in accelerator physics has raised much interest during the last decades, both in superconducting hadron colliders whose magnets have strong nonlinearities, and in high performance light sources. In this paper we review recent work in this field, limiting ourselves to the analysis of the single-particle dynamics in a strict sense, i.e., excluding beam-beam interaction, space charge phenomena, or intrabeam scattering.

We will initially make some remarks about the modeling of a lattice and the approaches to analyse the dynamics, namely numerical integration and perturbative theory (section 1). Then, we briefly outline the ideas that have been developed to speed up the numerical integration through fast symplectic tracking (section 2). Having built the tools for integrating the equations of motion, one can carry out simulations to evaluate the so-called dynamic aperture (DA), i.e. the dimension of the domain in phase space where trajectories are stable. We point out some difficulties in the DA definition in section 3. For electron machines the damping time is of the order of $10^{2}-10^{4}$ turns and therefore only the short-term stability is determinant. For large hadron machines the requested stability time is of the order of $10^{6}-10^{8}$ turns: in section 4 we review some methods that have been developed to analyse this problem and some numerical tools for the phase space analysis.

The leading mechanisms that rule both short term and long term dynamic aperture are not yet understood. Moreover, the parameteric dependence on the lattice lay-out is unknown with the exception of few cases. We make some basic remarks about this problem in section 5 . In section 6 we review some optimization techniques to increase the lattice dynamic aperture; we discuss the idea of replacing 
the optimization of the DA with the optimization of a quality factor (QF) that is strongly correlated with the DA. These techniques have been widely used in beam dynamics both for protons and electrons machines. The knowledge of a good QF allows a theoretical understanding of the phenomena that rule the DA and either an analytical optimization or a speeding up of the numerical optimization. Finally, the basic practical techniques that can be used to improve lattice performance (changing the optics, inserting correcting elements, sorting the magnets) are outlined in section 7 . Some open problems are given in section 8 .

\section{INTEGRATION OF EQUATIONS OF MOTION}

The single-particle motion in a magnetic lattice is described by an Hamiltonian with three degrees of freedom: two transversal $(x, y)$ and one longitudinal $s$. We restrict to the case of circular lattices, where the dependence on $s$ is periodic. It is customary to use $s$ as the independent time-like variable and therefore one ends with the Hamiltonian

$$
H\left(x, p_{x}, y, p_{y}, t, \delta ; s\right)
$$

that describes the single-particle motion; here $\delta$ is the relative deviation of the particle energy. The nonlinearities in $H$ can be summarized as follows

- In electron machines the main source of nonlinearities are the sextupoles that are used for chromatic correction, i.e., for reducing the change of the linear betatron frequencies for off-energy particles.

- In proton machines built with superconducting magnets the dipoles and the quadrupoles are affected by strong nonlinear errors.

Such a nonlinear Hamiltonian has no analytic solution and therefore one has to go for two complementary approaches.

- One can use numerical tools to provide an approximate solution to the equations of motion (tracking codes). Tracking is a precise tool to determine the particle trajectory in every regime (both weak and strong nonlinearities), but it has the drawback of being a 'black box' that provides little theoretical understanding of the nonlinear motion.

- One can use a perturbative approach to derive analytical information on some dynamical quantities such as detuning, phase space deformations, resonances, etc. Perturbative theory gives a worse approximation of motion compared to tracking, and fails when the nonlinearities become too strong; it has the advantage of providing an analytical understanding of the dynamics, even though much care has to be paid not to apply it beyond its validity limits.

It is general belief that the numerical integration should mantain the symplectic structure of the original Hamilton equations. This should be preserved also for 
electron machines, where the non-Hamiltonian effects due to the radiation should be added in the framework of a symplectic scheme.

A very simple method to derive an explicit symplectic integrator is the kick approximation. The idea is to replace a nonlinearity which is diffused along an element with one or more delta 'functions', keeping constant the integral of the nonlinearity along the element. This approach is widely used to track large hadron machines. More refined symplectic schemes can be worked out for general cases [1-3]. For short machines the problem of constructing explicit symplectic integrators is more involved [4], since for instance fringe fields at the edge of the magnets become relevant.

In any case, the motion along the lattice is integrated through a series of explicit symplectic nonlinear maps. These maps can be written using the powerful formalism of Lie series, whose first applications to accelerator physics date back to the second half of the seventies [5-7]. One can produce Taylor expansion of these maps; the map coefficients are related to the nonlinear aberrations of the lattice. By composing and truncating these maps one obtains the truncated one-turn map; truncation is unavoidable as computer codes can deal only with a limited number of Taylor coefficients (order 20 for a four-dimensional mapping is already very challenging in terms of memory and processing time). The formalism of truncated power series and its implementation in computer codes has been known for decades in the field of celestial mechanics [8,9], and applied to accelerator physics in the eighties (see for instance [10]).

A lot of analytical work has been carried out to describe betatron motion. One can choose between the standard approach based on canonical perturbative theory for hamiltonian flows $[11,12]$ and the discrete approach based on the truncated oneturn map and normal forms [13-15]. In the first case the perturbative parameters are usually the field gradients, whilst in the second one the series are in powers of the actions (i.e., amplitudes in phase space). This difference leads to a completely different ordering of the nonlinear contributions. We believe that the second approach in general is more suitable to describe the nonlinear motion. Moreover, effective codes have been written to compute the map perturbative expansion at arbitrary order $[14,16]$. This feature makes the map approach very attractive and powerful for the analysis of nonlinear betatron motion.

\section{FAST SYMPLECTIC TRACKING}

The numerical integration through symplectic tracking can be very onerous, es-

pecially in the case of large machines with several sources of nonlinearities. For hadron machines, this effect severely restricts the possibility of carrying out exhaustive studies of long-term stability. On the other hand, in the case of electron machines the dynamic aperture is determined by short-term phenomena, and the possibility of having fast tools to integrate the equation of motion allows a better optimization of the lattice parameters. 
For hadron machines the symplectic property is crucial, and therefore tracking the truncated one-turn map is not adequate, since it is symplectic only up to the truncation order. In order to speed up the integration keeping the symplecticity, one can use two methods

- Build an explicit symplectic map whose lower orders agree with the truncated one-turn map.

- Fit an explicit symplectic map to an extensive set of tracking data obtained with the standard procedures. Once this onerous operation is carried out, the map can be used instead of tracking.

An explicit symplectic map can be built using two different tools

- As a composition of a sequence of explicit nonlinear maps [17-19].

- Using a mixed variable generating function one can implicitly define a symplectic map. If there is a good initial guess for the map (for instance the truncated map is usually a very good first guess), using a Newton method one can easily invert the equation and obtain an explicit symplectic map [20].

The dynamics at low amplitudes is usually very well reproduced through the symplectified map. At high amplitudes the agreement can be worse, but one should point out that the tracking code itself is already an approximate solution to the equations of motion.

A crucial point for applications is to have a method that is really faster compared to tracking. One has to choose a compromise between the speed and the accuracy; indeed, it is not trivial to quantify the minimum degree of accuracy required. These comparison are not easy also because different tracking codes easily have rather different speeds, depending on platforms and code optimizations. We observe that whilst these methods have been widely applied in the U.S., in Europe they seem much less popular.

\section{DYNAMIC APERTURE (DA) EVALUATION}

"[Dynamic aperture] is one of the most fundamental and important objects in beam dynamics. [...] Even, it seems that there is no good constructive definition of dynamic aperture." This quotation [21] from the home page of the tracking code SAD (Strategic Accelerator Design), developed at KEK, well underlines the difficulties of the dynamic aperture issue. From the point of view of numerical simulation, the dynamic aperture $D(N)$ is a measure of the set of initial conditions in the $4 \mathrm{D}$ transverse phase space (plus the parametric dependence on off-momentum) that are stable for at least $N$ turns. It is rather surprising that not much effort has been developed to give a reasonable definition of DA and, in particular, to associate to the evaluated DA an error. The estimate of the error is crucial to check the validity of the DA improvements obtained by lattice optimization. 
The major difficulty in the DA definition is that the first amplitude where particle loss occurs at $N$ turns depends at least on three separate factors.

a) The first amplitude where particle loss occurs depends on the ratio of the linear invariants along which we start initial conditions. Usually one makes a radial scan

$$
x=A \cos \kappa \quad y=A \sin \kappa
$$

along a fixed $\kappa$ (usually $\kappa=\pi / 4$, i.e., $x=y$ ), and the initial momenta $\left(p_{x}, p_{y}\right)$ are set to zero. Indeed, according to $\kappa$ one has different linear invariants and therefore the whole dynamics is different (for instance the detuning strongly depends on the $\kappa$ ). This is due to the four-dimensionality of the problem.

b) The first amplitude where particle loss occurs depends on the initial phases in the two planes $\left(x, p_{x}\right)$ and $\left(y, p_{y}\right)$. This is due to the deformation of the orbits, which close to the dynamic aperture are not direct product of two rotations.

c) Particle losses take place in chaotic regions where one has sensitivity to initial conditions. Therefore, to each initial condition one cannot simply associate an escape time, but rather a distribution of possible escape times. This distribution can be rather wide (some orders of magnitudes). For instance in Fig. 1 we plot the distribution of the escape times for 50 particle started around a very tiny neighbourhood of a chaotic initial conditions for the Hénon map.

Due to these effects, the amplitude where particle loss occurs can vary in a range of $10-30 \%$ in non pathological cases. Moreover, the scan in phase space is discrete and this adds another source of error in the DA estimation.

One can outline two different strategies to overcome this problem:

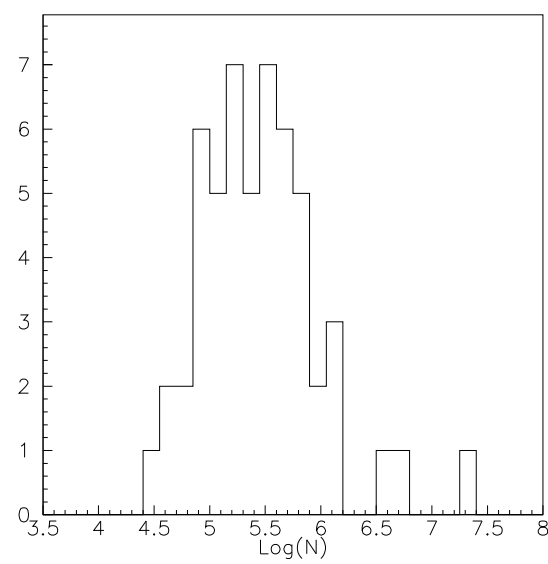

FIGURE 1. Escape times of 50 particles started around a chaotic initial condition at amplitude $\mathrm{x}=\mathrm{y}=0.47$ for the Hènon map, with linear frequencies 0.168 and 0.201 . 
- Fast but unprecise DA evaluation: one carries out tracking along one direction only, with one particle per initial condition (usually zero phases, and equal emittances). This is approach has been used for large machines where simulations are onerous. An estimate of the error associated to this DA evaluation is very hard to obtain, since the phase space dynamics has been analysed only in a very limited region.

- Averaging procedures: one carries out a richer and more onerous phase space sampling, and takes an average of the amplitude loss. In this way one can also give estimates of the error associated to this average. A procedure to take into account effects (a) and (b) has been given in [22], and error estimates have been given in [23]. One should associate to this dynamic aperture also a measure of the spread to provide a lower bound to the DA. In principle one could directly take the minimum amplitude over the phase space sampling, but this quantity is more unstable from a numerical point of view; moreover the error estimate is more troublesome.

\section{TRANSVERSE PHASE SPACE, LONG-TERM STABILITY, DIFFUSION}

\section{Transverse phase space tools}

During the last decade relevant advances have been carried out in the study of the phase space of $4 \mathrm{D}$ sympletic mappings that can model the transverse betatron motion. A major contribution has been given by frequency analysis tools [24] that through intensive tracking campaign allow to draw a complete picture of the global dynamics. Moreover, complementary analytical tools of resonant normal forms have been developed and arbitrary order codes which evaluate the resonance position and width are available [25].

In Ref. [24] a very precise numerical method has been presented to evaluate the nonlinear frequencies of time series. Moreover it has been shown that extensive tracking simulations over a two-dimensional grid of initial conditions in phase space (one typically sets the momenta to zero and scans along the physical coordinates) allow to give a numerical reconstruction of global dynamics in phase space. For each initial condition one evaluates the frequencies with high precision (at least $10^{-5}-10^{-6}$ ); less than $10^{4}$ are sufficient to get such a precision - see also [26] for a review of the methods and estimates of their errors. Then one can draw the results either in the frequency or in the action space.

- Tune footprint: the image of the uniform grid of initial conditions is plotted in the space of frequencies, and its deformation allows to see what are the strong resonances (see [24,27] for more details). 
- Action print or resonance net: we plot in $(x, y)$ only those initial conditions whose orbits are resonant; i.e., whose nonlinear frequencies satify a resonant condition up a given order. In this way one obtains in the physical space a picture of the resonant channels, and, contrary to the footprint, the width of the resonance is directly visible in the graph. More details on this method can be found in $[28]$.

For instance in Fig. 2 we show the resonance net of a toy model (Hènon map plus an octupole). One can see that the main resonances that affect the motion are $(1,-1)$ and $(1,-4)$. The linear tune is set on 0.28 and 0.31 . The short-term dynamic aperture is between 0.4 and 0.5 arbitrary units. One can see the relation of the resonances with the dynamic aperture. The plot also shows a wide net of high order resonances that cross each other also at very low amplitudes. The origin itself is resonant since the linear frequencies satisfy a 12 th order resonant condition $(8,4,1)$. Chaotic bands are also visible using this method (see [28]).

Unfortunately, most tracking codes are not yet equipped with automatic procedures to carry out simulations on a two-dimensional grid of initial conditions. It also would be a nice improvement to carry out the scan over the linear invariants and not over the coordinates, i.e. $\left(x^{2}, y^{2}\right)$ instead of $(x, y)$, as proposed in [24]; this

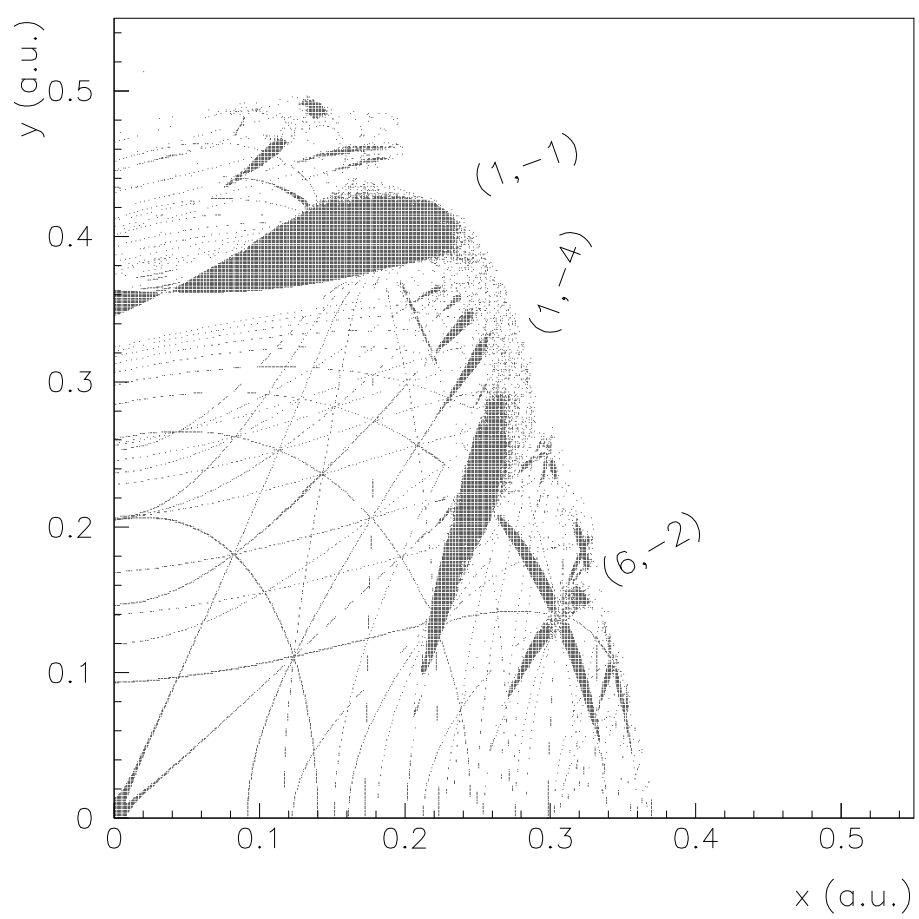

FIGURE 2. Initial conditions that give rise to resonant orbits (in black) for the 4D Hénon map plus an octupole, through tracking and frequency analysis. Initial momenta set to zero. 
scan is more natural, and the grid is denser at high amplitudes, that are the more interesting for the dynamics.

The reconstruction of the net of resonances in the space of initial conditions can be realized also through the perturbative tools of normal forms. Running a resonant normal form for each resonance, one can evaluate the resonance position and width through semi-analytical tools, and obtain a perturbative reconstruction of the dynamics in phase space. As for all perturbative tools, results close to dynamic aperture can be not accurate, and one has to pay much attention to the truncation order. The advantage is that analytical minimization of resonances can be worked out, since all the parametric dependence can be included. More details can be found in [25].

\section{Methods for long-term stability estimates}

Sophisticated tools have been developed to evaluate the long-term stability. One can group them in three main areas.

\section{Bounds on invariants}

All stable orbits have two nonlinear invariants that are constant along the orbit, for instance the actions or the frequencies. On the other hand, invariants are not defined for the unstable orbits. If a method to evaluate the nonlinear invariants with infinite precision from tracking data would be available, one could easily distinguish stable from unstable orbits by evaluating the presence of a drift in the nonlinear invariants. For unstable particles, this drift can be extrapolated to longer times to get a stability estimate: fixing an outer domain where one assumes that a fast diffusion is present (short term dynamic aperture), one can compute the time necessary to the particle to go from the initial condition to this outer domain.

Therefore, in order to have realistic estimates, a method to evaluate the nonlinear invariants from tracking data with the highest precision must be worked out: in fact, any numerical error in the determination of the invariant will be erroneously considered as a diffusion in phase space.

The main source of error for most methods is the same: island structures associated to resonances. For instance, the invariant defined through nonresonant normal forms is very imprecise on resonances, and therefore it overestimates the diffusion in these regions: indeed, most resonances are stable at sufficiently low amplitudes. A similar phenomenon occurs if we consider the variation of the frequencies along the same orbit: on resonant orbits every algorithm that provides frequencies has a much larger error, and therefore using this method the diffusion is considerably overestimated along resonances.

A very elaborate and clever numerical method to construct a nonlinear invariant based on interpolating the action with splines has been proposed in $[29,30]$, and has 
led to some reasonable estimates of stability times that have been verified through tracking [31].

\section{Particle loss predictors}

Also in this case one considers dynamical variables that can be extracted from short-term tracking data, as the variation of the actions or of the frequencies $[27,28]$ along the orbit, or the rate of divergence of two nearby orbits [28,32,23]. The difference with respect to the previous approach is that one fixes a threshold, and assumes that all the particles whose orbits produce an indicator above the threshold will be lost and all the other ones are stable. No information is given on the time necessary to lose the stability.

The Lyapunov exponent has been very popular in celestial mechanics, and has also been used in accelerator physics. Results show that one can detect the border of instability using short term tracking data when no tune modulation is present; in the case of tune modulation the method does not seem to be effective [23].

\section{Survival plots and DA extrapolation}

A simple and effective way to plot the information contained in tracking data is given by survival plots, where the stability time is plotted versus the amplitude in phase space. Usually tracking is carried along one direction, i.e. equal emittances and zero phases, with very dense scan in the amplitude (see for instance [33]): these plots should show the trend of long-term dynamic aperture, but are usually rather irregular, and it seems rather difficult to work out a trend for times larger than the tracked ones.

An improvement of survival plots can be obtained if a dense scan along the amplitude in one direction is substituted by several, less dense scans along different ratio of emittances $[28,23]$ : if we plot the averaged amplitude versus the stability time it turns out that it is well-interpolated by the three-parameter formula

$$
D(N)=A+\frac{B}{\log ^{\kappa} N}
$$

The formula has been shown to work for a wide variety of models which range from the 4D Hènon map to the LHC with tune modulation (see Fig. 3). The quantity $A$ represents the dynamic aperture extrapolation for 'infinite times', and becomes negative when the tune modulation is increased. The exponent $\kappa$ is arond 1.5 for the purely $4 \mathrm{D}$ case and decreases when the tune modulation becomes more relevant.

This formula gives on the one hand a clear model of the averaged phase space dynamics, providing a rate for the stability times which has the same logarithmic 
dependence on the time as the Nekhoroshev estimate. Moreover, it can be extrapolated to predict long-term dynamic aperture. For cases with tune modulation one can extrapolate of one-two order of magnitudes in the number of turns [23,34].

\section{Diffusion}

The possibility of fitting the dynamics of particle loss with a diffusion process, according to the ideas developed in [35], have been discussed and analysed for a long time. Both numerical simulations and experiments have been carried out, several times including multifrequency modulation of the linear frequencies that model tune ripple. Experimental data obtained at FNAL have been analysed in the framework of the diffusion equation [36]. In [37] the influence of two ripples on a simplified lattice model (Hènon map) has been analysed, using the concept of sidebands and overlapping. The effectiveness of the ripple correction to improve beam lifetime in the HERA has been shown in [38]. Different mechanisms that lead to transverse proton diffusion have been analysed in [39], where a diffusion equation and resonance parameters evaluated through perturbative theory were used to understand which mechanism could explain diffusion in the HERA. Experiments carried out at the SPS are reported in [40]. On the theoretical side, interesting results have been obtained by using the Neihstadt adiabatic theory [41]: for a twodimensional phase space with a single resonance and a periodic modulation of the linear frequency, it has been proved that the process can be described in terms of

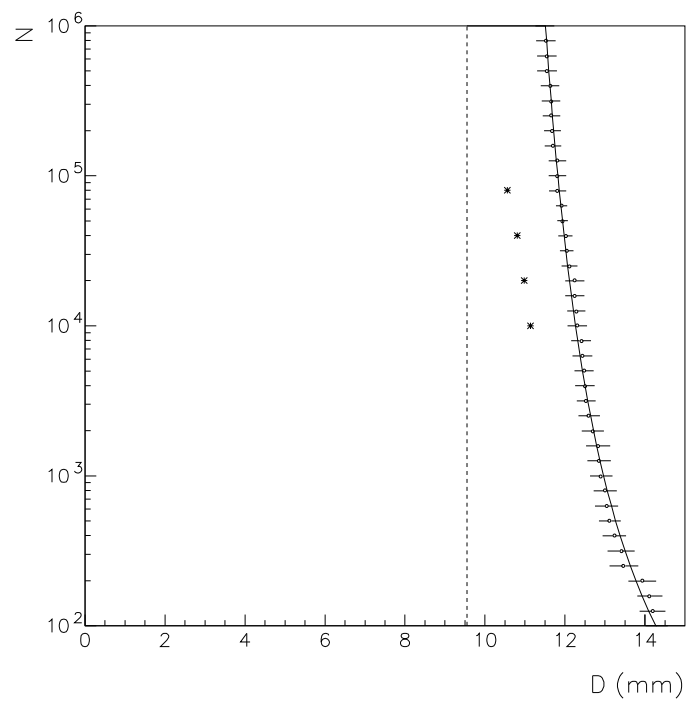

FIGURE 3. Averaged Dynamic Aperture versus stability time (survival plot) for an LHC model with imperfections, off momentum. Bars: tracking data. Solid line: interpolation. Circles: Lyapunov prediction. 
a random walk in the action space [42].

In our opinion, notwithstanding the considerable amount of work that has been carried out during the last decade, we are far from a complete understanding of these phenomena. In particular, we point out that a good example of agreement between tracking simulations of dynamics at high amplitudes and a diffusion equation has not been reached, even in the simplest case of purely four-dimensional dynamics.

In fact, some authors did not believe in the possibility of using a diffusion equation to describe long-term dynamics [43]. This clearly depends also on the mechanisms that are involved in the long-term losses.

Chirikov theory of overlapping treats the case of a main resonance and of its sidebands created by tune modulation or other mechanisms. Indeed, in the general case of several resonances in a four-dimensional phase space, that cross each other also at low amplitudes (see Fig. 2), the Chirikov idea of overlapping becomes hard to apply. What is sure is that wide chaotic bands are a very relevant mechanism, but the origin of these bands is still unclear.

\section{DA VS LATTICE PARAMETERS}

The dynamic aperture depends on both linear (tunes, beta functions, phase advances, linear coupling, chromaticity ...) and nonlinear (multipolar errors, multipolar correctors, detuning, nonlinear chromaticity, ...) parameters. We limit ourselves to a few simple remarks.

- Lattice dominated by a multipole. If the dynamic aperture is dominated by the effect of a single multipole, one can derive a simple scaling that provides the dependence of the DA versus the multipole strength and versus the beta function where the multipole is located.

- DA versus fractional linear tune. Even for a simple model such as a linear lattice plus a sextupole, the dependence on the linear tune is extremely complex and is given by the intricate relation between resonances, detuning and nonlinearities (see Fig. 4). Only in the case of a linear tune close to an unstable resonance (for example, resonance $(3,0)$ or $(1,2)$ for a lattice with a normal sextupole) one can give an analytical estimate of the DA. In fact, in this case the stability is lost on the separatrix of the unstable resonance that can be evaluated through resonant perturbative theory [44]. In the generic case the situation is more complex and a general well known rule is to avoid to set the linear tune close to excited low order resonances (i.e., resonances from 3 to 5 , see for instance [45]). Nevertheless high order resonances can be relevant to the dynamics (see for instance the case of resonance $(7,0)$ in the LHC [46]). For electron machines, extensive studies of the DA as a function of the fractional tunes have been carried out using fast tracking (see for instance the so-called 'swamp plots' in [47]. 
- DA versus integer part of the tune. The integer part of the tune can be very relevant since it determines the phases between the nonlinear elements of the machine, leading to coherent sum or partial compensation. The integer part of the tune is not present in the linear part of the one-turn map (that contains only the fractional part), but its value determines the strength of the higher order map coefficients. Analytical approaches to the optimization of the integer part of the tune can be built through the perturbative theory based on hamiltonian flows [48].

\section{QUALITY FACTORS AND CORRELATIONS}

Since in the generic case the dependence of the DA on the lattice parameters is unknown, one has to use numerical integration (i.e., tracking) to evaluate the DA and to optimize it. Indeed, since many years the accelerator physicists community has widely used quality factors (QF) to understand and improve lattice performances.

- Definition and characteristics. A QF is a function of the dynamical variables (trajectory in phase space, nonlinear frequencies ...) and/or of the lattice parameters (multipoles, linear optics, ...). It can be either analytical, i.e. can be derived directly from the one-turn map coefficients, or numerical, i.e. evaluated through the postprocessing of a set of tracking data. The QF must have two fundamental characteristics: it must be fast to compute (compared to the DA) and for the set of considered different lattices it must have a good correlation with the DA.

- Examples of QFs. The nonlinear aberrations (i.e., some map coefficients; the norm of the map (i.e., a weigthed sum of map coefficients up to the truncation order); the tuneshift (either evaluated through perturbative series or computed

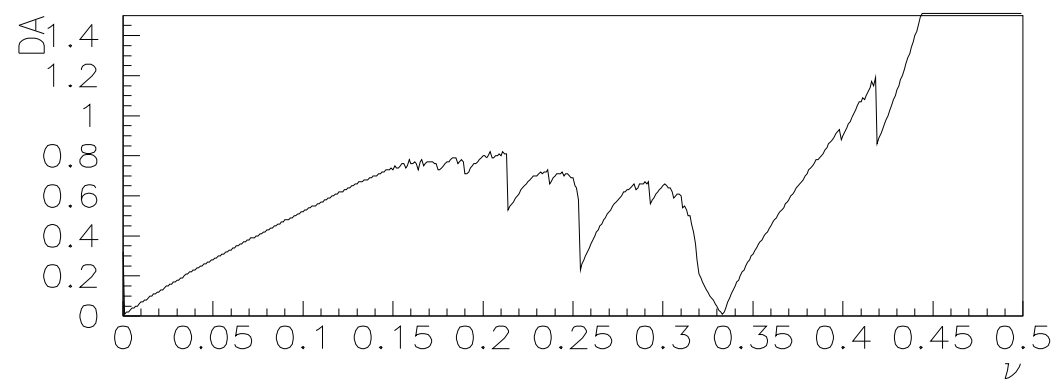

FIGURE 4. Dynamic aperture at 10000 turns for the 2D Hénon mapping versus the fractional part of the linear tune. 
using tracking data); the 'resonances' (there are several ways to extract QF related to resonances, both numerical and perturbative); the smear, i.e. the deformation of the orbits with respect to the linear case [46]; the fixed points residuals [49].

- Correlation between QF and DA. Even though in several works the QF is established a priori, we believe that one should always check that the given QF is correlated with the DA, since the QF is model-dependent. For instance, in some lattices the detuning can be the driving mechanism of the DA and therefore is a good QF to choose, whilst for other cases it can be irrelevant. The correlation between QF ad DA cannot be established a priori with the present knowledge.

- What a QF can provide. Once a correlation between the QF and the DA has been shown through tracking a number of cases, one can use the QF instead of the DA for carrying out more onerous simulations, such as a wider statistical analysis of the lattice or of the lattice parameters. With this respect, the QF is a tool that allows to save CPU time. Another very relevant feature is that the QF gives an insight on the mechanism that rules DA and therefore can suggest analytical ways of carrying out optimizations [46,50]. Even though one must spend some CPU time to check the QF correlation with the DA, one obtains a better theoretical understanding of the lattice dynamics. In this respect, the QF approach is useful also for electron machines where the need of $\mathrm{CPU}$ time is not so stringent as for proton machines.

- Some applications. The minimization of the detuning has been used nearly one decade ago to correct the systematic errors in the SSC and in the LHC [51,52]. The correction of resonances has been used as criterion to sort the dipoles to compensate random errors [50,53]. QF related to the resonances have been also used at PEP-II to improve the lattice [47] and for the LHC to determine the lattice performances [46].

\section{WAYS OF CORRECTING}

The ways of optimizing a lattice can be summarized as follows:

- Changing the linear parameters of the lattice, that is the optics: tune, beta functions, position of the magnetic elements ...

- Inserting in the lattice some nonlinear elements that reduce the nonlinearity already present in the lattice. We distinguish between three main approaches:

- Local correction: the corrector element is close to the error source. This is the case for instance of the sextupolar chromatic correctors in a regular lattice put close to the regular quadrupoles, which are the sources of chromaticity. 
- Lumped correction: corrector elements not too far from the error source; this was used for the SSC and the LHC lattices when only two correctors per half cell were used to effectively reduce the nonlinearities originated by four dipoles $[51,52,54]$.

- Global correction: only a few correctors are inserted, distant from the error sources.

Evidently, the last solution is the least expensive but it is rarely feasible. Also in the case of the local correction, there is a limit in the nonlinearity strength beyond which the correction becomes uneffective.

- Sorting the magnets: this method does not involve the construction of new hardware. If several magnets with the same function and hardware characteristics (such as for instance the cell dipoles) feature large random errors, one can install the magnets in a sequence that minimizes the nonlinear effects. The problem is very hard since one has to analyse a huge number of possible permutations. To apply this procedure one must measure all the magnets, and have some space to store a large batch of magnets. Several studies have been carried out for different machines [50,53,55-58].

\section{SOME OPEN PROBLEMS}

We conclude this overview by pointing out some topics that we believe are open problems in this field.

- About long-term stability: it is still not clear what the mechanism is that creates macroscopic chaotic bands and how resonances are related to it. Moreover, more work should be done in order to understand whether the loss of integrability in phase space is a sudden phenomenon or whether it is gradual.

- About dynamic aperture: is it necessary to go towards a probabilistic definition of the DA, i.e. asking that not all particles survive for a given time but that a very small percentage could escape? This question is related to the solution of the previous one about the loss of integrability.

- About diffusion: does the diffusion equation really fit experimental or numerical data?

- About quality factors: is it possible to know a priori (i.e., without tracking) whether a given QF is correlated with the dynamic aperture ?

- About correction: when is local correction necessary and when is global correction sufficient?

- About experiments: what informations on nonlinear motion can we extract from experiments ? Good agreement on detuning has been reached (see for 
instance [59]). Some work has been carried out on measuring the map coefficients in experiments, and also to obtain experimentally a tune footprint (see for instance [60,61]). Both tasks are very challenging, the main aim being a more complete modelization of the machine and a better undestranding of the dynamic aperture.

\section{ACKNOWLEDGEMENTS}

We wish to thank M. Cornacchia and C. Pellegrini for the kind invitation to the conference. We are very indebted to Prof. Turchetti and to W. Scandale for introducing us to this subject and for their constant help and support. We wish to thank M. Giovannozzi and for critical reading of the manuscript. This work is dedicated to A. della Monica.

\section{REFERENCES}

1. Forest, E., and Ruth, D., Physica D 43, 105-17 (1990).

2. Yoshida, H., Phys. Lett. A 150, 262-8 (1990).

3. Forest, E., Bengtsson, J., and Reusch, M., Phys. Lett. A 158, 99-101 (1991).

4. Forest, E., Reusch, M., Bruhwiler, D., and Amiry, A., Part. Accel. 45, 65-94 (1994).

5. Dragt, A., and Finn, J. M., J. Math. Phys. 17, 2649-60 (1976).

6. Dragt, A., and Finn, J. M., J. Math. Phys. 20, 2649-60 (1979).

7. Dragt, A., Nucl. Instrum. and Methods Phys. Res., A 258, 339-354 (1987).

Perturbative theory for betatron motion

8. Giorgilli, A., Comp. Phys. Comm. 16, 331 (1979).

9. Servizi, G., and Turchetti, G., Comput. Phys. Commun. 32, 201-7 (1984).

10. Berz, M., Part. Accel. 24, 109 (1989).

11. Schoch, A., CERN 57-21 (1957).

12. Guignard, G., CERN 76-06 (1976).

13. Bazzani, A., Mazzanti, P., Servizi, G., and Turchetti, G., Nuovo Cim. B 102, 51-80 (1988).

14. Berz, M., Irwin, J., and Forest, E., Part. Accel. 24, 109 (1989).

15. Bazzani, A., Todesco, E., Turchetti, G., and Servizi, G., CERN 94-02 (1994).

16. Bazzani, A., Giovannozzi, M., and Todesco, E., Comp. Phys. Commun. 86, 199-207 (1995).

Fast symplectic tracking

17. Rangarajan, G., Dragt, A., and Neri, F., Part. Accel. 28, 119-24 (1990).

18. Gjaja, I., Part. Accel. 43, 133-44 (1994).

19. Shi, J., and Yan, Y., Phys. Rev. E 48, 3943-51 (1993).

20. Berg, J. S., Warnock, R. L., Ruth, R. D., and Forest, E., Phys. Rev. E 49, 722-739 (1994).

Dynamic aperture definition

21. SAD web pages http://www-acc-theory.kek.jp/sad/tracking.html 
22. Todesco, E., and Giovannozzi, M., Phys. Rev. E 53, 4067 (1996).

23. Giovannozzi, M., Scandale, W., and Todesco, E., Phys. Rev. E 57, 3432-43 (1998). Transverse phase space, long-term stability, diffusion

24. Laskar, J., Physica D 67, 257-81 (1992).

25. Todesco, E., Gemmi, M., and Giovannozzi, M., Comp. Phys. Comm. 106, 169-80 (1997).

26. Bazzani, A., et al., Part. Accel. 52, 147-77 (1996).

27. Laskar, J., Froeschlé, C., and Celletti, A., Physica D 56, 253-69 (1992).

28. Giovannozzi, M., Scandale, W., and Todesco, E., Part. Accel. 56, 195-225 (1997).

29. Warnock, R. L., Phys. Lett. 66, 1803-6 (1991).

30. Warnock, R. L., and Ruth, R. D., Physica D 56, 188-215 (1992).

31. Warnock, R. L., and Berg, J. S., AIP Conf. Proc. 395, 423-45 (1996).

32. Schmidt, F., Willeke, F., and Zimmermann, F., Part. Accel. 35, 249-256 (1991).

33. Yan, Y., SSC 500 (1991).

34. Böge, M., and Schmidt, F., AIP Conf. Proc. 405, 201-210 (1997).

35. Chirikov, B. V., Phys. Rep. 52-5, 263-379 (1979).

36. Chen, T., et al., Phys. Rev. Lett. 68, 33-6 (1992).

37. Brüning, O., Part. Accel. 41, 133-51 (1993).

38. Brüning, O., and Willeke, F., Part. Accel. 54, 237-46 (1996).

39. Zimmermann, F., Part. Accel. 49, 67-104 (1995).

40. Fischer, W., Giovannozzi, M., and Schmidt, F., Phys. Rev. E 55, 3507-20 (1997).

41. Neishtadt, A., Sov. J. Plasma Phys. 12, 568-73 (1986).

42. Bazzani, A., Brini, F., and Turchetti, G., AIP Conf. Proc. 395, 129-38 (1997).

43. Gerasimov, A., CERN-SL (AP) 92-38 (1992).

DA vs. lattice parameters

44. Giovannozzi, M., Phys. Rev. E 53, 6403 (1996).

45. Robin, D., and Laskar, J., Part. Accel. 54, 193-202 (1995).

46. Papaphiloppou, Y., and Schmidt, F., these proceedings.

47. Yan, Y., Irwin, J., and Chen, T., Part. Accel. 55, 17-26 (1996).

48. Talman, R., LHC Project report 197 (1998).

QF and lattice optimization

49. Wan, W., Cary, J. R., and Shasharina, S. G., AIP Conf. Proc. 395, 407-422 (1996).

50. Giovannozzi, M., Grassi, R., Scandale, W., and Todesco, E., Phys. Rev. E 52, 3093101 (1995).

51. Neuffer, D., Part. Accel. 27, 209-14 (1990).

52. Scandale, W., Schmidt, F., and Todesco, E., Part. Accel. 35, 53-88 (1991).

53. Willeke, F., DESY HERA 87-12 (1987).

54. Neuffer, D., and Forest, E., Phys. Lett. A 135, 197-201 (1989).

55. Gluckstern, R. L., and Ohnuma, S., IEEE Trans. Nucl. Sci. NS-32, 2314-16 (1985).

56. Ziemann, V., Part. Accel. 51, 155-79 (1995).

57. Shi, J., and Ohnuma, S., Part. Accel. 56, 227-47 (1997).

58. Bartolini, R., et al., Nuovo Cim. B 113, 511 (1998).

59. Gareyte, J., Scandale, W., and Schmidt, F., IOP Conf. Series 131, 235-48 (1993).

60. Irwin, J., AIP Conf. Proc. 326, 662 (1995).

61. Terebilo, A., et al, these proceedings. 